\title{
Erratum to: Cutaneous Complications of Targeted Melanoma Therapy
}

Emily de Golian ${ }^{1}$

Bernice Y. Kwong ${ }^{1}$

Susan M. Swetter ${ }^{1,2}$

Silvina B. Pugliese ${ }^{1, *}$

\section{Address}

${ }^{*}, 1$ Department of Dermatology, Cutaneous Oncology, Stanford University Medical Center and Cancer Institute, 780 Welch Road, CJ220F, Palo Alto, CA, 94304-5779, USA

Email: spugliese@stanford.edu

${ }^{2}$ Dermatology Service, Veterans Affairs Palo Alto Health Care System, Palo Alto, CA, USA

Published online: 5 November 2016

(C) Springer Science+Business Media New York 2016

Erratum to: Curr Treat Options in Oncol (2016) 17:57

DOI 10.1007/s11864-016-0434-0

In the original version of this article, which published in Current Treatment Options in Oncology, Volume 17, Issue 11, November 2016, the drug "cobimetinib" was erroneously referred to as "cometinib" throughout the text. 